\title{
Challenges in skeletal muscle physiology
}

\author{
Peter J. Reiser* \\ Division of Oral Biology, The Ohio State University, Columbus, OH, USA \\ *Correspondence: reiser.17@osu.edu
}

Skeletal muscle subserves physiological functions that require the generation of force and/or movement. This abundant tissue is extraordinarily heterogeneous with respect to the expression of a multitude of protein isoforms (e.g., Bottinelli, 2001) to meet the broad diversity in demands for motor functions, from rapid and extremely precise eye rotations, to track quickly moving objects, often with unpredictable trajectories, to slow and or even sustained postural adjustments, such as with attempts to blend with the environment during episodes of predator avoidance. Much has been learned about molecular and cellular mechanisms that underlie muscle force generation and shortening since the writing of The Canon of Medicine, a compilation of Greek and Roman medicine by the Persian physician and philosopher, Avicenna, in the eleventh century (Shah, 1966). Avicenna provided an anatomical description of human skeletal muscles, with references to function. Considering the more contemporary status of the understanding of skeletal muscle, models of the crossbridge cycle were proposed in the twentieth century and have since been further tested and refined, and continue to provide a solid framework for new and sustained investigations directed toward what remain as many vexing questions. Intricacies of excitation-contraction coupling have been studied extensively for several decades, as has diversity in muscle protein expression, both at the level of gene expression and with respect to protein isoform patterns in different fiber types. Skeletal muscle has been, and continues to be, intensely studied, and much has been learned about regulation of activation, the crossbridge cycle, relaxation, and alterations associated with skeletal muscle disease and aging. However, numerous challenges remain.

Major gaps exist in our understanding of fundamental mechanisms underlying contraction, as well as of mechanisms that contribute to the control of muscle physiological properties. The central role of calcium ions in muscle activation, that assures efficient excitation-contraction coupling, has been very extensively examined, yet controversy persists concerning how extracellular calcium ions, calcium stores in the sarcoplasmic reticulum, associated calcium-transport and release mechanisms, and calcium-binding proteins interact to achieve activation and relaxation in a consistent manner in skeletal muscle (e.g., Beam and Bannister, 2010). Vigorous investigation in this area is perpetuated, due, in part, to new controversies that evolve as even greater complexity in calcium-handling mechanisms is realized.

Extremely elaborate studies, from a technical perspective, have been conducted, with resolution that was virtually unimaginable even a couple of decades ago, to decipher conformational changes in specific structural domains of single myosin molecules expressed in skeletal, cardiac, and smooth muscle, as well as unconventional myosins in non-muscle cells, using in vitro motility assays and optical trap approaches (see Sung et al., 2010, for a review of some of the relevant literature). Potentially important interactions between myosin and other thick-filament proteins and thin filament proteins, and interactions between filaments such as those involving the thick-filament based myosin binding protein-C (MyBP-C) with actin, remain partially unresolved. For example, evidence has accrued to support a role for an interaction between MyBP-C and myosin in modulating skeletal and cardiac muscle contraction (e.g., Hofmann et al., 1991a,b; Luther et al., 2008). More recently, it has been reported that interactions of MyBP-C with actin could also have a modulatory role in contraction in cardiac muscle (Shaffer et al., 2009; Shchepkin et al., 2010) and this raises the possibility of a related mechanism in skeletal muscle. There is also a very recent report of evidence for multiple isoforms of slow-type MyBP-C in skeletal muscle (Ackermann and Kontrogianni-Konstantopoulos, 2010); so it is clear that much remains to be learned in this area, especially the physiological significance of the full range of interactions within and between thick and thin filament proteins. An ultimate challenge is to develop a comprehensive model of conformational changes of thick and thin filament proteins and of intermolecular interactions underlying activation, as well as force generation and shortening, in skeletal muscle with high temporal and spatial resolution.

The potential for the expression of a vast array of contractile protein isoforms is very well appreciated and gene regulatory mechanisms for many of these proteins have been described. However, the mechanisms that regulate/coordinate the expression of the multitude of thick and thin filament protein isoforms, such that only a finite set of isoform programs actually exist (e.g., Bicer and Reiser, 2009) among populations of muscle fibers, are not well understood. Epigenetic and miRNA mediated mechanisms are now recognized as being critically important in regulating protein expression in skeletal muscle. Much remains to be explored to determine the scope of these mechanisms and, very importantly, to identify miRNA targets to more fully understand control of protein expression and, thereby, regulation of physiological properties in skeletal muscle.

It is well recognized that activation of skeletal muscle is a highly cooperative process, involving not only calcium ions binding to troponin-C, but also dynamic interactions among thin filament proteins and between thick and thin filament proteins, resulting in an orchestrated mechanism with high fidelity that results in rapid and efficient activation and relaxation. However, many aspects of the actual mechanisms that normally underlie cooperativity during skeletal muscle activation are not well understood (see review by Fitzsimons and Moss, 2007).

There are several other sarcomeric proteins for which roles in the regulation of contraction have been proposed, including the very large proteins nebulin and titin that 
span considerable distances in sarcomeres. Roles for determining primarily passive mechanical properties or aspects of sarcomeric structure have been proposed for both of these proteins, but there is also evidence suggesting that these proteins may have more active roles in determining contractile properties of cardiac muscle (e.g., Cazorla and de Tombe, 2008; Greaser et al., 2008), and, potentially, of skeletal muscle.

Profound decrements in motor functions occur during the progression of several myopathies and during the normal course of aging. Significant progress has been made in identifying and understanding mechanisms that contribute to these pathophysiological changes, but this is an area of practical importance that remains poorly understood, especially considering the impacts on critical motor functions in a significant portion of the human population.

Clearly, several important challenges exist in the field of skeletal muscle physiology. A high level of optimism that these challenges will be met is well justified, given the success that has resulted from intense efforts and incredibly ingenious approaches employed in the past in many laboratories throughout the world. What is perhaps most important is that we can look forward to addressing these and many other areas and, at the same time, identifying what are currently unanticipated additional frontiers in skeletal muscle physiology.

\section{REFERENCES}

Ackermann, M.A., and Kontrogianni-Konstantopoulos, A. (2010). Myosin binding protein-C slow: an intricate subfamily of proteins. J. Biomed. Biotechnol.2010, Article ID 652065.

Beam, K. G., and Bannister, R. A. (2010). Looking for answers to EC coupling's persistent questions. J. Gen. Physiol. 36, 7-12.

Bicer, S., and Reiser, P.J. (2009). Myosin isoform expression in dog rectus muscles: patterns in global and orbital layers and among single fibers. Invest. Ophthalmol. Vis. Sci. 50, 157-167.

Bottinelli, R. (2001). Functional heterogeneity of mammalian single muscle fibres: do myosin isoforms tell the whole story? Pflugers Arch. 443, 6-17.

Cazorla, O., and de Tombe, P. P. (2008). Some rat: a very special rat with a rather special titin. J. Mol. Cell. Cardiol. 44, 976-978.

Fitzsimons, D. P., and Moss, R. L. (2007). Cooperativity in the regulation of force and the kinetics of force development in heart and skeletal muscles: cross-bridge activation of force. Adv. Exp. Med. Biol. 592, 177-189.

Greaser, M. L., Warren, C. M., Esbona, K., Guo, W. Duan, Y., Parrish, A. M., Krzesinski, P. R., Norman, H. S., Dunning, S., Fitzsimons, D. P., and Moss, R. L. (2008). Mutation that dramatically alters rat titin isoform expression and cardiomyocyte passive tension. J. Mol. Cell. Cardiol. 44, 983-991.

Hofmann, P. A., Greaser, M. L., and Moss, R. L. (1991a). C-protein limits shortening velocity of rabbit skeletal muscle fibres at low levels of $\mathrm{Ca}^{2+}$ activation. J. Physiol. 439, 701-715.

Hofmann, P. A, Hartzell, H. C., and Moss, R. L. (1991b). Alterations in $\mathrm{Ca}^{2+}$ sensitive tension due to partial extraction of C-protein from rat skinned cardiac myocytes and rabbit skeletal muscle fibers. J. Gen. Physiol. 97, 1141-1163.

Luther, P. K., Bennett, P. M., Knupp, C., Craig, R., Padrón, R., Harris, S. P., Patel, J., and Moss, R. L. (2008). Understanding the organisation and role of myosin binding protein $\mathrm{C}$ in normal striated muscle by comparison with MyBP-C knockout cardiac muscle. J. Mol. Biol. 384, 60-72.

Shaffer, J. F., Kensler, R. W., and Harris, S. P. (2009). The myosin-binding protein $\mathrm{C}$ motif binds to F-actin in a phosphorylation-sensitive manner. J. Biol. Chem. 284, 12318-12327.

Shah, M. H. (1966). The General Principles of Avicenna's Canon of Medicine. Karachi: Inter Services Press.

Shchepkin, D. V., Kopylova, G. V., Nikitina, L. V., Katsnelson, L. B., and Bershitsky, S. Y. (2010). Effects of cardiac myosin binding protein-C on the regulation of interaction of cardiac myosin with thin filament in an in vitro motility assay. Biochem. Biophys. Res. Commun. 401, 159-163.

Sung, J., Sivaramakrishnan, S., Dunn, A. R., and Spudich, J.A. (2010). Single-molecule dual-beam optical trap analysis of protein structure and function. Meth. Enzymol. 475, 321-375.

Received: 21 September 2010; accepted: 28 October 2010; published online: 22 November 2010.

Citation: Reiser PJ (2010) Challenges in skeletal muscle physiology. Front. Physio. 1:151. doi: 10.3389/ fphys.2010.00151

This article was submitted to Frontiers in Striated Muscle Physiology, a specialty of Frontiers in Physiology.

Copyright (c) 2010 Reiser. This is an open-access article subject to an exclusive license agreement between the authors and the Frontiers Research Foundation, which permits unrestricted use, distribution, and reproduction in any medium, provided the original authors and source are credited. 